\title{
Otimização da colônia de formigas aplicada ao problema da programação e roteirização de veículos para o transporte de pessoas portadoras de deficiência
}

\author{
Cristina Mayumi Baba (EEM) mayumi vr@yahoo.com.br \\ Fernanda Henriques Martins Corrêa (EEM) fefa correa@yahoo.com.br \\ Sabrina Alessandra Cordeiro de Souza (EEM) souzasabrina@hotmail.com \\ Thais Mandel Wahba (EEM) wahbinha@uol.com.br \\ Afonso Celso Medina (EEM) medina@maua.br
}

\section{Resumo}

Este trabalho propõe uma heurística de solução para o problema da programação e roteirização de veículos para o transporte de pessoas portadoras de deficiência. Neste tipo de problema, conhecido na literatura como "problema do dial-a-ride", os usuários fazem solicitações de transporte de um ponto especifico de embarque para um ponto especifico de desembarque. O objetivo do problema é determinar uma programação de rotas que atenda às solicitações de transportes sujeita às restrições de número de veículos disponíveis, janelas de tempo nos pontos de coleta e entrega, capacidade do veículo, precedência da coleta sobre a entrega e tempo máximo de tolerância do passageiro dentro do veículo. O problema estudado neste artigo aplica-se ao caso em que a frota de veículos é finita, heterogênea e os veículos partem de diferentes garagens dispostas geograficamente na região de onde surgem as solicitações. A heurística proposta é baseada na meta-heurística da colônia de formigas e procura maximizar o número de solicitações atendidas ao menor custo possível. Os resultados computacionais, obtidos a partir da aplicação da heurística em dados reais de um operador da cidade de Sorocaba-SP, sugerem um desempenho promissor para a utilização da otimização da colônia de formigas no problema do dial-a-ride.

Palavras Chave: Transporte de pessoas portadoras de deficiência, Meta-heurística da colonia de formigas, Problema do dial-a-ride.

\section{Introdução}

Este trabalho trata da programação e roteirização de veículos para o transporte de pessoas portadoras de algum tipo de deficiência e com uma grande dificuldade de mobilidade pessoas com alto grau de dependência ou que não apresentam condições de locomoção com autonomia nos meios de transportes coletivos convencionais. Esse tipo de serviço é geralmente operado por veículos adaptados com elevadores ou rampas de acesso, e com espaço reservado para o transporte de uma ou mais cadeiras de rodas. Os usuários fazem solicitações de serviço indicando: o ponto de embarque, o horário desejado do embarque, o número de pessoas que serão embarcadas, a utilização ou não de cadeira de rodas, o ponto de desembarque e o horário desejado do desembarque. Cabe ao operador do sistema programar roteiros para atendimento dessas solicitações utilizando-se dos veículos disponíveis em garagens distribuídas na região de onde partem as solicitações. Trata-se, portanto, de um problema de coleta e entrega com restrições de janela de tempo, capacidade do veículo e tempo máximo de viagem - entendido aqui como o tempo máximo que o usuário tolera permanecer no veículo. Problemas dessa natureza são conhecidos na literatura como o problema do dial-a-ride.

Como o número de veículos é finito, o objetivo do problema é construir uma programação de roteiros de modo a maximizar o número de solicitações atendidas, minimizando o custo de 
atendimento, pois nem sempre será possível atender a todas as solicitações. Considera-se que o custo fixo de um novo veículo é sempre muito superior ao custo de deslocamento desse veículo. Assim, uma solução que atende a um mesmo número de requisições, mas utiliza um veículo a menos que outra, será sempre considerada melhor. Por outro lado, entre soluções que atendem o mesmo número de solicitações e utilizam o mesmo número de veículos, será considerada melhor aquela em que a distância total percorrida pelos veículos for menor.

Baba et al. (2003) apresentam uma relação de prefeituras do estado de São Paulo que possuem esse tipo de sistema de transporte. Os autores observam que esses municípios não conseguem atender a toda demanda de usuários devido não só a limitação de recursos financeiros e de veículos disponíveis, mas também devido à falta de um sistema computacional eficiente para a construção desses roteiros.

Este artigo está dividido em 6 seções. A seção 2 apresenta uma breve revisão bibliográfica do problema do dial-a-ride. A seção 3 relata algumas estratégias de solução para o problema apresentado e a seção 4 descreve a abordagem proposta baseada na colônia de formigas. A seção 5 apresenta os resultados computacionais obtidos e a seção 6 é reservada às conclusões e sugestões para trabalhos futuros.

\section{Revisão Bibliográfica}

Existem diversos trabalhos publicados sobre o problema do transporte de pessoas portadoras de deficiência. Os trabalhos de Savelsbergh e Sol (1995), Toth e Vigo (1997) e Madsen et al. (1995) servem como um ponto de partida para o estudo do problema. Notadamente, o problema do dial-a-ride é uma generalização do problema da coleta e entrega com restrição de janela de tempo que, por sua vez, tem sua origem no problema clássico do caixeiro viajante.

O problema pode ser categorizado em duas classes distintas (SAVELSBERGH e SOL, 1995): dinâmicos e estáticos. Os problemas de natureza dinâmica são aqueles em que apenas parte das requisições de serviço é conhecida ao iniciar-se o processo de construção dos roteiros novas solicitações podem surgir após a conclusão do procedimento de programação de rotas e devem ser inseridas nas rotas já existentes ou novas rotas devem ser criadas. Nos problemas de natureza estática todas as solicitações de serviço são previamente conhecidas dentro do horizonte de tempo do estudo.

O problema do dial-a-ride aplicado ao transporte de pessoas portadoras de deficiência possui algumas particularidades que aumentam o seu grau de dificuldade quando comparado a outros problemas de roteirização: a acomodação dos passageiros dentro do veículo é diferenciada pela eventual presença de usuários em cadeiras de rodas, alguns usuários necessitam da presença obrigatória de um acompanhante e o usuário é geralmente mais sensível à duração da viagem.

Jaw et al. (1986) propõem uma heurística de inserção seqüencial. Neste tipo de heurística, uma nova rota só pode ser construída quando a anterior estiver completa, gerando assim uma rota completa por interação da heurística. O trabalho de Madsen et al. (1995) é uma aplicação do problema ao transporte de idosos e deficientes da cidade de Copenhagen na Dinamarca. $\mathrm{O}$ sistema desenvolvido é capaz de atender cerca de 300 solicitações diariamente de forma dinâmica. A heurística proposta é derivada da heurística de inserção seqüencial de Jaw et al. (1986). O trabalho de Toth e Vigo (1997), aplicado ao problema do transporte de deficientes da cidade de Bologna na Itália, é baseado na idéia da inserção paralela: um conjunto inicial de rotas é estabelecido e as solicitações são inseridas nesse conjunto inicial. Caso não seja mais possível a inserção das solicitações nas rotas já existentes, uma nova rota é criada e o processo continua até que todas as solicitações tenham sido atendidas. Caso não existam veículos 
suficientes para o atendimento de todas as requisições, o sistema prevê que veículos extras ou táxis são utilizados. Terminada a fase de construção de rotas, a heurística realiza um processo de melhoria baseado na busca tabu.

O trabalho de Znamensky (2000) estuda o problema aplicado ao serviço ATENDE da prefeitura de São Paulo-SP. O autor estuda duas abordagens para o problema: a solução exata por meio de um modelo de programação inteira e uma heurística de inserção paralela baseada no trabalho de Madsen et al. (1995), acrescida de um procedimento de melhoria local que apresenta resultados satisfatórios para o caso em estudo.

\section{Estratégias de Solução}

As estratégias de solução para o problema do dial-a-ride podem ser classificadas em métodos exatos e métodos heurísticos.

Os métodos exatos procuram obter a solução ótima para o problema, a partir da construção de modelos matemáticos de otimização, cuja solução ótima pode ser obtida por meio de algoritmos conhecidos. Contudo, dada a complexidade do problema do dial-a-ride, as soluções obtidas são geralmente restritas às instâncias mais simples do problema. Sutcliffe e Board (1990), por exemplo, apresentam um modelo de programação inteira, resolvido por meio do algoritmo branch-and-bound, para o transporte de deficientes para um único centro de reabilitação, que se mostrou ineficaz na solução de instâncias reais do problema. Psaraftis (1980) aplica a programação dinâmica para resolver um problema do tipo dial-a-ride com apenas um veículo e sem restrições de janela de tempo. Nos testes computacionais realizados pelo autor, o tempo de processamento aumenta rapidamente com o aumento do número de solicitações, inviabilizando o uso do método para problemas reais.

Os métodos heurísticos são aqueles que permitem a obtenção de soluções viáveis, não necessariamente ótimas, mas com uma boa aproximação para problemas reais e com maior rapidez que os métodos exatos. Dentre os métodos heurísticos utilizados para o problema, destacam-se os trabalhos de Jaw et al. (1986), Madsen et al. (1995) e Toth e Vigo (1997).

Neste trabalho adotou-se um método heurístico composto de duas fases: criação de um conjunto de rotas iniciais e inserção das solicitações às rotas. A primeira fase foi baseada na heurística de inserção paralela proposta por Toth e Vigo (1997), e a segunda fase utiliza a meta-heurística da colônia de formigas.

\section{Criação das rotas iniciais e aplicação da otimização da colônia de formigas}

Este trabalho aplica a meta-heurística da colônia de formigas proposta por Dorigo e Caro (1999), que é um desenvolvimento natural dos algoritmos de formigas propostos por Dorigo (1992) para a solução de problemas de otimização combinatória, como o problema do caixeiro viajante.

As formigas são insetos sociais que possuem um sistema complexo de organização e divisão de tarefas, cuja principal função é garantir a sobrevivência do formigueiro. Chama atenção a complexidade das tarefas executadas pelo formigueiro quando comparada com a simplicidade de cada formiga individualmente. A meta-heurística da colônia de formigas foi inspirada na observação das colônias de formigas reais, em particular em como elas encontram o menor caminho entre a fonte de alimentos e o formigueiro.

Para a obtenção do alimento para o formigueiro, a colônia resolve um interessante problema de otimização. Inicialmente, as formigas percorrem de modo aleatório a região próxima ao formigueiro em busca do alimento. Cada formiga, enquanto percorre o seu caminho, deposita sobre o solo uma substância chamada feromônio, formando um caminho ou rastro de feromônio. As formigas subseqüentes detectam o a presença desta substância e tendem a 
escolher o caminho marcado com a maior concentração de feromônio. O feromônio portanto, além de possibilitar a formação de um caminho de volta para a formiga, também tem a função de informar as outras formigas sobre quais os melhores caminhos até o alimento. Depois de algum tempo, os caminhos mais eficientes - ou de menor distância percorrida até o alimento acumulam uma quantidade maior de feromônio. Inversamente, os caminhos menos eficientes - ou de maior distância percorrida até o alimento - apresentam uma pequena concentração de feromônio, devido ao menor número de formigas que passaram por ele e ao processo de evaporação natural do feromônio. No problema de otimização que o formigueiro se defronta, cada formiga é capaz de construir uma solução completa do problema; contudo, a melhor solução só é obtida mediante cruzamento das diversas soluções encontradas.

O Sistema de Formigas, primeira meta-heurística de otimização de colônia de formigas proposta por Dorigo (1992), quando aplicado ao problema do caixeiro viajante, inicia-se com cada formiga construindo uma solução a partir de um dos nós da rede do problema. Cada formiga $k$ constrói o seu caminho movendo-se através de uma seqüência de locais vizinhos, onde os movimentos são selecionados segundo uma distribuição de probabilidades dada por:

$$
p_{i j}^{k}= \begin{cases}\frac{\left[\tau_{i l}\right] \cdot\left[\eta_{i l}\right]^{\beta}}{\sum_{l \in J_{i}^{k}}\left[\tau_{i l}\right] \cdot\left[\eta_{i l}\right]^{\beta}} & \text { se } j \in J_{i}^{k} \\ 0 & \text { caso contrário }\end{cases}
$$

Onde:

$p_{i j}^{k}=$ probabilidade da formiga $k$, que se encontra em $i$, escolher o nó $j$ como próximo nó a ser visitado;

$\tau_{i j}=$ quantidade de feromônio existente no arco $(i, j)$. Inicialmente, adota-se um mesmo valor $\tau_{0}$ para todos os arcos da rede;

$\eta_{i j}=$ função heurística que representa a atratividade do arco $(i, j)$. No caso do problema do caixeiro viajante, adota-se o inverso do valor da distância entre os nós $i$ e $j$;

$J_{i}^{k}=$ conjunto de pontos ainda não visitados pela formiga $k$, que se encontra atualmente no ponto $i$;

$\beta$ = valor heuristicamente escolhido, que pondera a importância da quantidade de feromônio existente no arco em relação à distância entre os nós $i$ e $j$.

A expressão (1) mostra que a preferência da formiga por determinado caminho é maior para os caminhos com mais feromônio e com menor distância.

Após um certo número $m$ de formigas terem finalizado suas rotas, a quantidade de feromônio é atualizada em cada arco de modo a reforçar o caminho obtido pela melhor formiga. Assim, para cada arco $(i, j)$ da rede, adiciona-se uma quantidade de feromônio proporcional ao tamanho da rota obtida:

$$
\tau_{i j}=(1-\rho) \tau_{i j}+\rho \Delta \tau_{i j}^{m e l h o r}
$$

$$
\Delta \tau_{i j}^{\text {melhor }}= \begin{cases}\frac{1}{L_{\text {melhor }}} & \text { se }(i, j) \text { pertence a rota construída pela melhor formiga da iteração } \\ 0 & \text { caso contrário }\end{cases}
$$

O primeiro termo da equação (2) é responsável pela evaporação do feromônio, onde $\rho$ é um parâmetro que determina a velocidade da evaporação. O segundo termo é responsável por 
aumentar a concentração de feromônio apenas nos arcos visitados pela melhor formiga. $L_{\text {melhor }}$ é a distância total percorrida na rota construída pela melhor formiga da iteração quanto menor a rota maior a quantidade de feromônio depositada.

O procedimento se repete até que um número máximo de iterações tenha sido alcançado ou caso não se verifiquem mais melhorias nas soluções obtidas.

Diversas melhorias ou alterações na otimização do Sistema de Formigas podem ser realizadas. O procedimento Max-Min, proposto por Hoos e Stützle (2000), impõe que nenhum arco tenha uma quantidade de feromônio superior a $\tau_{\text {máx }}$ ou inferior a $\tau_{\min }$. Estes parâmetros são definidos a cada iteração da heurística e procuram minimizar a possibilidade da solução convergir para soluções locais muito rapidamente, garantindo uma maior exploração dos arcos pelas formigas.

A estratégia de solução proposta neste trabalho para o problema do dial-a-ride é ligeiramente diferente da descrita anteriormente para o problema do caixeiro viajante. As formigas não constroem rotas caminhando de uma solicitação a outra, mas sim atribuindo solicitações aos veículos ou rotas. Deste modo, o procedimento guarda uma maior semelhança com o proposto por Ducatelle e Levine (2001) para o problema do empacotamento binário.

A escolha do significado do feromônio é fundamental para o sucesso da heurística e para a própria compreensão do procedimento otimizador. No caso em estudo, o feromônio representa o quanto é favorável que a solicitação $i$ seja atribuída ao veículo $j$. Considerando-se $r$ veículos e $n$ solicitações, a matriz de feromônios $\tau_{i j}$ é do tipo $r \times n$. Escolheu-se esta forma de representação do feromônio por dois motivos: a sua relação direta com o procedimento de construção de rotas de Toth e Vigo (1997) e a dificuldade de se construir rotas à semelhança do problema do caixeiro viajante, pois, no caso do problema em estudo, a inserção de um novo nó na rota significa, de fato, a inserção de dois pontos simultâneos (um de coleta e outro de entrega) que geralmente alteram a seqüência de pontos visitados da rota.

$\mathrm{O}$ valor adotado para a função $\eta_{i j}$ é o inverso do custo de inserção da solicitação $j$ na rota $i$, sendo este custo calculado como proposto por Toth e Vigo (1997). Outras escolhas para o significado do feromônio e da função $\eta_{i j}$ são possíveis e são objeto de estudo de uma pesquisa atualmente em desenvolvimento por um dos autores. Caracterizado o feromônio e a função $\eta_{i j}, p_{i j}^{k}$ representa a probabilidade da formiga $k$ inserir a solicitação $j$ no veículo $i$. A atualização do feromônio ao final de cada iteração continua sendo realizada como o indicado pelas expressões (2) e (3), contudo, o valor $L_{\text {melhor }}$, anteriormente a distância total percorrida, agora é dado pela expressão:

$$
L_{\text {melhor }}=\frac{n_{v} \cdot d}{N}
$$

Na expressão (4), $N$ é o número de solicitações inseridas, $n_{v}$ é o número de veículos utilizados na solução e $d$ é a distância total percorrida na solução encontrada. A expressão (4) quando associada à expressão (3) leva a heurística a depositar mais feromônio nas soluções que atendem mais solicitações, utilizam menos veículos e percorrem distâncias menores.

A seguir, descreve-se resumidamente o procedimento heurístico proposto. Para um maior detalhamento, consultar Baba et al. (2003).

\section{$1^{a}$ Fase: Determinação das rotas iniciais}

Esta fase é baseada no procedimento de Toth e Vigo (1997). Para uma dada fração de solicitações, o procedimento inicia-se pela determinação do número mínimo de veículos 
necessários para o atendimento desta fração. Os veículos, ordenados por suas eficiências operacionais, são então atribuídos às solicitações, de modo que para cada veículo é construída uma rota que possui apenas uma solicitação. Resumidamente, temos o seguinte procedimento:

Passo 1 - Calcular a eficiência de cada veículo de acordo com suas respectivas características operacionais e ordená-los do mais eficiente para o menos eficiente.

Passo 2 - Calcular o número de veículos necessários para atender uma fração pré-estabelecida das solicitações.

Passo 3 - Classificar em ordem decrescente o grau de dificuldade de cada solicitação requisitada.

Passo 4 - Designar uma solicitação para cada um dos veículos selecionados por meio de um cálculo de atratividade que considera: a proximidade de cada solicitação e a distância média entre a solicitação a ser inserida e as solicitações já inseridas em cada um dos veículos anteriores.

\section{$2^{\text {a }}$ Fase: Inserção das viagens não roteirizadas}

Nesta fase ocorre a atribuição das solicitações ainda não roteirizadas às rotas existentes e a criação de novas rotas quando é inviável a inserção de uma solicitação nas rotas já existentes. Inicialmente, uma quantidade de feromônio $\tau_{0}$ é depositada em cada par $(i, j)$, rota-viagem. Um conjunto de $m$ formigas é utilizado em cada interação. Para cada uma das formigas, o procedimento tem os seguintes passos:

Passo 1 - Construir a matriz de custos de inserção rota-viagem, onde as linhas representam as rotas existentes e as colunas representam as solicitações ainda não roteirizadas.

Passo 2 - Para cada solicitação, deve-se:

2.1.Calcular a probabilidade de inserção desta solicitação em cada uma das rotas já existentes, segundo a expressão (1);

2.2.Inserir segundo a distribuição de probabilidades construída no passo anterior, uma solicitação a uma rota;

\subsection{Recalcular a matriz de custos.}

Passo 3 - Calcular os limites $\tau_{\text {máx }}$ e $\tau_{\text {min }}$ de acordo com o sistema de formigas Max-Min (STÜTZLE e HOOS, 2000).

Passo 4 - Depositar uma nova quantidade de feromônio nas rotas encontradas pela melhor formiga da iteração, utilizando as expressões (2), (3) e (4).

O procedimento descrito é repetido até um número máximo de iterações permitidas, ou caso a solução não apresente mais melhora entre uma iteração e outra.

\section{Resultados Computacionais}

O procedimento descrito na seção anterior foi aplicado ao serviço prestado pela Empresa de Desenvolvimento Urbano e Social de Sorocaba - URBES, no município de Sorocaba-SP. Atualmente, este serviço é programado de forma manual. Neste estudo considera-se um dia de serviço com 311 solicitações requisitadas para uma frota de 12 veículos.

A frota disponível é composta por 6 microônibus com 24 assentos normais e capacidade para uma cadeira de rodas, 4 microônibus com rampa de acesso, podendo alocar 5 cadeiras de 
rodas e 10 assentos normais e 2 vans com rampa de acesso, contendo 4 assentos e 1 local para cadeira de rodas.

As características das solicitações se resumiam em 229 usuários de assentos normais, 82 usuários que permaneciam nas cadeiras de rodas e 84 que necessitavam de acompanhantes.

O procedimento foi implementado em Delphi 6.0 da Borland Software Corporation. Os testes forma realizados em um microcomputador Pentium IV com 1,6 Ghz e $256 \mathrm{Mb}$ de memória RAM. Diversos parâmetros de configuração foram testados e a melhor configuração encontrada da colônia foi com 20 formigas. O número de iterações foi limitado em 150 iterações. A tabela 1 apresenta algumas características da solução obtida.

\begin{tabular}{lc}
\hline \multicolumn{1}{c}{ Parâmetro de Saída } & Valores \\
\hline Requisições atendidas & 205 \\
Requisições não atendidas & 106 \\
Rotas iniciais & 5 \\
Veículos utilizados & 12 \\
Veículos não utilizados & 0 \\
Tempo de processamento (minutos) & 30 \\
\hline
\end{tabular}

Tabela 1 - Parâmetros e resultados

Pode-se notar que mais da metade dos usuários é beneficiada pelo serviço, ou seja, aproximadamente $66 \%$ das viagens requisitadas são atendidas. Contudo, a solução gerou rotas que concentram as viagens em períodos específicos (manhã, tarde ou noite), tornando os veículos ociosos e mal distribuídos ao longo do dia. Isto ocorre devido às restrições de tempo máximo de viagem e de janela de tempo, pois o conjunto de solicitações do município de Sorocaba tem um perfil concentrado em períodos, como indicado na figura 1. Visando melhorar a qualidade da solução, isto é, aumentar o número de viagens atendidas por veículo, as solicitações fornecidas foram agrupadas em três períodos de acordo com seus horários desejados de entrega, antes da aplicação da heurística, conseguindo assim, atender ao conjunto completo de solicitações - para maiores detalhes, consultar Baba et al (2003). Essa prática também é realizada no município de Sorocaba. O procedimento manual adotado pela URBES leva, em média, dois dias de trabalho.

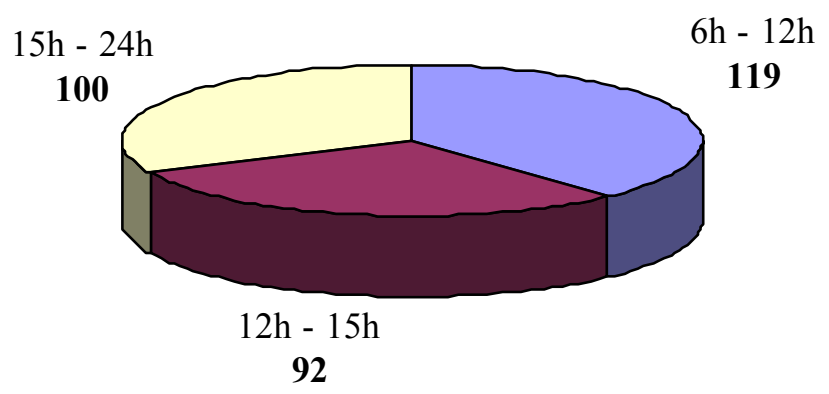

Figura 1 - Concentração das requisições nos períodos manhã, tarde ou noite. 


\section{Conclusões e Sugestões para Trabalhos Futuros}

Neste trabalho apresentou-se uma aplicação da meta-heurística da colônia de formigas para o problema da programação e roteirização de veículos para o transporte de pessoas portadoras de deficiência.

Até onde a pesquisa bibliográfica atingiu, esse é o primeiro trabalho a utilizar a metaheurística da colônia de formigas especificamente para esta categoria de problemas. $\mathrm{O}$ procedimento proposto foi baseado na heurística de inserção paralela proposta por Toth e Vigo (1997) e na meta-heurística da colônia de formigas proposta por Dorigo e Caro (1999). Os resultados computacionais obtidos em um problema teste construído a partir de dados reais mostraram um resultado promissor para o uso da meta-heurística.

Esta é uma pesquisa exploratória do potencial da meta-heurística. Novas formas de representação do feromônio e de construção de rotas são desenvolvimentos naturais deste trabalho. Atualmente, um dos autores está desenvolvendo uma pesquisa na direção de uma nova metodologia para solução do problema, que inclui o aspecto dinâmico dos sistemas de transporte especiais, não considerado neste trabalho.

\section{Referências}

BABA, C. M.; CORRÊA F. H. M.; SOUZA, S. A. C. \& WAHBA, T. M. (2003). Programação e roteirização de veículos para o transporte de pessoas portadoras de deficiência. Trabalho de Graduação, Centro Universitário do Instituto Mauá de Tecnologia - CEUN-IMT, São Caetano do Sul, 102 p.

DORIGO, M. \& CARO, G. DI (1999) - The Ant Colony Optimization Meta-Heuristic. In D. Corne, M. Dorigo and F. Glover, editors, New Ideas in Optimization, McGraw-Hill, p.11-32.

DORIGO, M. (1992) - Optimization, Learning and Natural Algorithms. Ph.D.Thesis, Politecnico di Milano, Italy.

DUCATELLE, F. \& LEVINE, J. (2001) - Ant colony optimisation and local search for bin packing and cutting stock problems. Proceedings of the UK Workshop on Computational Intelligence (UKCI-01) Edinburgh.

JAW, J. J., ODONI, A. R., PSARAFTIS, H. N. \& WILSON, N. H. M. (1986) - A heuristic algorithm for the multi vehicle advance request dial-a-ride problem with time windows. Transportation Research, Vol. 20B, n.3, p.243-257.

MADSEN, O. B. G.; RAVAN, H. F. \& RYGAARD, J. M. (1995) - A heuristic algorithm for a dial-a-ride problem with time window, multiple capacities, and multiple objectives. Annals of Operational Research, n.60, p. 193-208.

PSARAFTIS, H. N. (1980) - A Dynamic Programming Solution to the Single-Vehicle Many-to-Many Immediate-Request Dial-A-Ride Problem. Transportation Science Vol. 14, n.2, p.130-154.

SAVELSBERGH, M \& SOL, M. (1995) - The general pickup and delivery problem. Transportation Science, v.29, n.1, p.17-29.

STÜTZLE, T. \& HOOS, H. (2000). MAX-MIN ant system. Future Generation Computer Systems, Vol. 16, n.8, p.889-914.

SUTICLIFFE, C. \& BOARD, J (1990) - Optimal Solution of a Vehicle-routeing Problem: Transporting Mentally Handicapped Adults to an Adult Training Centre. Journal of the Operational Research Society, Vol. 41, n.1, p. 61-67.

TOTH, P. \& VIGO, D. (1997) - Heuristic algorithm for the handicapped person transportation problem. Transportation Science, Vol. 31, n.1, p. 60-71.

ZNAMENSKY, A. (2000) - Um modelo para roteirização e programação do transporte de deficientes, Dissertação (Mestrado) - EPUSP, Departamento de Engenharia de Transportes, São Paulo, 144 p. 